\title{
Cardiac hypertrophy and histone deacetylase-dependent transcriptional repression mediated by the atypical homeodomain protein Hop
}

\author{
Hyun Kook, ${ }^{1}$ John J. Lepore, ${ }^{1}$ Aaron D. Gitler, ${ }^{1}$ Min Min Lu, ${ }^{1}$ Wendy Wing-Man Yung, ${ }^{2}$ \\ Joel Mackay, ${ }^{2}$ Rong Zhou, ${ }^{3}$ Victor Ferrari, ${ }^{1}$ Peter Gruber, ${ }^{4}$ and Jonathan A. Epstein ${ }^{1}$ \\ ${ }^{1}$ Cardiovascular Division, University of Pennsylvania Health System, Philadelphia, Pennsylvania, USA \\ ${ }^{2}$ School of Molecular and Microbial Biosciences, University of Sydney, Sydney, New South Wales, Australia \\ ${ }^{3}$ Department of Radiology, University of Pennsylvania, Philadelphia, Pennsylvania, USA \\ ${ }^{4}$ Department of Surgery, Children's Hospital of Philadelphia, Philadelphia, Pennsylvania, USA
}

\begin{abstract}
Activation of multiple pathways is associated with cardiac hypertrophy and heart failure. Repression of antihypertrophic pathways has rarely been demonstrated to cause cardiac hypertrophy in vivo. Hop is an unusual homeodomain protein that is expressed by embryonic and postnatal cardiac myocytes. Unlike other homeodomain proteins, Hop does not bind DNA. Rather, it modulates cardiac growth and proliferation by inhibiting the transcriptional activity of serum response factor (SRF) in cardiomyocytes. Here we show that Hop can inhibit SRF-dependent transcriptional activation by recruiting histone deacetylase (HDAC) activity and can form a complex that includes HDAC2. Transgenic mice that overexpress Hop develop severe cardiac hypertrophy, cardiac fibrosis, and premature death. A mutant form of Hop, which does not recruit HDAC activity, does not induce hypertrophy. Treatment of Hop transgenic mice with trichostatin A, an HDAC inhibitor, prevents hypertrophy. In addition, trichostatin A also attenuates hypertrophy induced by infusion of isoproterenol. Thus, chromatin remodeling and repression of otherwise active transcriptional processes can result in hypertrophy and heart failure, and this process can be blocked with chemical HDAC inhibitors.
\end{abstract}

J. Clin. Invest. 112:863-871 (2003). doi:10.1172/JCI200319137.

\section{Introduction}

Cardiac hypertrophy can occur in response to normal physiological stimuli or may be maladaptive leading to cardiac dilatation and congestive heart failure (1). Transcriptional regulatory pathways that control homeostasis of cardiac myocyte size are complex and poorly understood. In the perinatal period, cardiac myocytes largely stop dividing, and further cardiac growth is associated with myocyte hypertrophy. Pathologic hypertrophy is accompanied by reactivation of fetal gene programs that may be triggered by alterations in calcium homeostasis and mechanical stretch with resultant transcriptional activation (2-4). Excessive hypertrophy often leads to cardiac dilation and congestive heart failure, which is the leading hospital discharge diagnosis in the United States (5).

Received for publication June 5, 2003, and accepted in revised form July 15, 2003.

Address correspondence to: Jonathan A. Epstein, 954 Biomedical Research Building II, 421 Curie Boulevard, Philadelphia, Pennsylvania 19104, USA. Phone: (215) 898-8731; Fax: (215) 573-2094; E-mail: epsteinj@mail.med.upenn.edu. Conflict of interest: The authors have declared that no conflict of interest exists.

Nonstandard abbreviations used: nuclear factor of activated T cells (NFAT); histone deacetylase (HDAC); myocyte enhancer factor 2C (MEF2C); serum response factor (SRF); smooth muscle $22 \alpha(\mathrm{SM} 22 \alpha)$; atrial natriuretic factor (ANF); trichostatin A (TSA); prostate specific antigen (PSA).
The genetic programs leading to cardiac hypertrophy are diverse and complex. Mutations in a growing number of genes encoding structural components of the contractile unit, the sarcomere, result in hypertrophic cardiomyopathy in humans and in animal models (6). Intracellular signaling pathways stimulated by $\beta$-adrenergic agonists (7) and intracellular stretch-sensing mechanisms (8) also induce hypertrophic gene programs. These stimuli frequently involve alterations in calcium homeostasis and result in the activation of prohypertrophic transcriptional programs $(2,9)$. One pathway that has been suggested to mediate calciumtriggered transcriptional activation involves the calcium-binding protein calcineurin, which functions to dephosphorylate nuclear factor of activated $\mathrm{T}$ cells (NFAT), resulting in nuclear translocation and transcriptional activation of prohypertrophic genes (10). An alternative pathway involves a putative calcium-sensitive kinase that phosphorylates class II histone deacetylases (HDACs), resulting in their association with 14-3-3 molecules, dissociation from myocyte enhancer factor $2 \mathrm{C}(\mathrm{MEF} 2 \mathrm{C})$, and translocation out of the nucleus $(11,12)$. This results in derepression of MEF2C and activation of prohypertrophic genes. Hence, class II HDACs have been postulated to repress cardiac hypertrophy.

Histone acetylation, mediated by histone acetyl transferases, is a mechanism for modifying the structure of 
chromatin such that nucleosomes are relaxed, resulting in transcriptional activation. The converse reaction is mediated by HDACs, which induce deacetylation, chromatin condensation, and transcriptional repression (13-15). At least nine mammalian HDAC genes have been described that fall into two categories: class I and class II. A third class homologous to the yeast protein Sir2 have also been described. In addition to a domain exhibiting deacetylase activity, class II HDACs contain an amino-terminal region capable of mediating proteinprotein interactions. Chemical inhibitors of HDAC activity have been identified and synthesized, and recent studies indicate a potential role for these compounds in promoting differentiation of some myeloid tumors, suggesting their possible use as anticancer agents (16). These agents are surprisingly well tolerated, and numerous phase I and phase II trials are underway $(17,18)$. The effects of HDAC inhibitors on cardiac myocyte homeostasis in vivo have not been described.

Recently, we and others identified an unusual regulator of cardiac-specific gene transcription $(19,20)$. Hop encodes a 73-amino acid protein that includes a 60 -amino acid motif homologous to the homeodomain of Hox transcription factors. Unlike Hox homeodomains, however, Hop is lacking certain conserved amino acid residues that are required for proteinDNA interactions, and Hop is unable to bind DNA. Nevertheless, Hop is a nuclear protein that can function to modulate transcription. Hop can physically associate with serum response factor (SRF) and inhibits the ability of SRF complexes to activate transcription of cardiac-specific genes $(19,20)$. For instance, forced expression of SRF and myocardin can result in transactivation of smooth muscle $22 \alpha$ (SM22 $\alpha$ ) and atrial natriuretic factor (ANF) promoters. Addition of Hop inhibits this activation. In Hop-deficient hearts, SRF-dependent genes are upregulated. Hop is expressed by cardiac myocytes during gestation and in the adult. In the absence of Hop, neonatal mice display enhanced cardiac myocyte proliferative indices and an increased number of cardiac myocytes (20). Hence, Hop is thought to modulate the delicate balance between cardiac replication and differentiation. The role of Hop in the adult myocyte remains unknown.

\section{Methods}

Transgenic mice. A cDNA encoding a $3^{\prime}$ hemagglutinin-tagged Hop was subcloned into SalI-HindIII sites

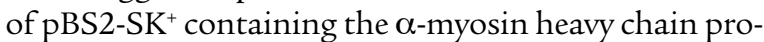
moter (Genbank no. U71441) (a gift from J. Robbins, Children's Hospital of Cincinnati, Cincinnati, Ohio, USA) (21). NotI-digested plasmid was injected into fertilized oocytes. For HopH2 mice, $\alpha$-MHC-Hop plasmid was subjected to site-directed mutagenesis (Stratagene, La Jolla, California, USA), resulting in the replacement of LIAAE with AAASM in the second helix of Hop (19). Three-week old Hop transgenic or wild-type littermates, or mice treated with isoproterenol infusion, were administered $0.6 \mathrm{mg} / \mathrm{kg}$ trichostatin A (TSA)
(Sigma-Aldrich, St. Louis, Missouri, USA) by intraperitoneal injection daily for 14 days. Alternatively, sodium valproate $0.71 \% \mathrm{wt} / \mathrm{vol}$ (Sigma-Aldrich) was added to drinking water available ad libitum. CD1 mice were treated with isoproterenol $(30 \mathrm{mg} / \mathrm{kg} /$ day) or saline by implantation of an osmotic minipump (Alzet 1002; Durect Corp., Cupertino, California, USA) as recommended. Histology protocols are available at http:// www.uphs.upenn.edu/mcrc.

Echocardiogram and MRI. Mice were anesthetized with a $1 \%$ isoflurane, temperature was maintained at $37^{\circ} \mathrm{C} \pm 0.1^{\circ} \mathrm{C}$, and mice were imaged using a 4.7-tesla horizontal-bore spectrometer using an elliptical surface coil. ECG-gated cine images were acquired as described (22). Echocardiography was performed using a $15-\mathrm{MHz}$ transducer and an Acuson Sequoia C256 machine.

Invasive bemodynamics. Hop ${ }^{-/-}$mice 40-45 weeks old $(n=10)$ were compared with wild-type littermates $(n=12)$, and 6-week-old Hop transgenic mice $(n=6)$ were compared with wild-type littermates $(n=6)$. Anesthesia was induced with $3 \%$ isoflurane and maintained by ventilation with $0.75 \%$ isoflurane. Temperature was maintained at $37^{\circ} \mathrm{C}$. A 1.4 French micromanometer catheter (Micro-Tip SPR-671; Millar Instruments, Houston, Texas, USA) was inserted via the right carotid artery, transduced, and recorded using PowerLab/16 SP (ADInstruments Pty Ltd., Colorado Springs, Colorado, USA). Isoproterenol (40 $\mathrm{ng} / \mathrm{kg}$ in PBS) was injected directly into the left internal jugular vein. An unpaired Student's $t$ test was used to compare hemodynamic variables between groups.

Cell culture, constructs, and transfection. Cos, 293T, and $10 \mathrm{~T} 1 / 2$ cells were maintained in DMEM with $10 \%$ FBS (Invitrogen Life Technologies, Carlsbad, California, USA) and transfected using FuGene6 (Roche Diagnostics Corp., Indianapolis, Indiana, USA). After 24 hours, 10-100 nmol/1 TSA (Sigma-Aldrich), or $2 \mu \mathrm{g} / \mathrm{ml} \mathrm{Scrip-}$ taid, or its inactive negative control, Nullscript (BioMol Research Laboratories Inc., Plymouth Meeting, Pennsylvania, USA), was added and incubated for 24 hours. Transfection protocol, SM22 $\alpha$-luciferase, pcDNA3myocardin, pcDNA3.1-Hopwt-myc, and pcDNA3.1HopH2-myc constructs were described previously (19). HDAC9 (AF332492) was amplified from the embryonic day 13.5 (E13.5) heart cDNA library (CLONTECH Laboratories Inc., Palo Alto, California, USA) and subcloned in pcDNA3.1 V5/His TOPO TA expression vector (Invitrogen Life Technologies).

Circular dichroism. Samples were dissolved in buffer containing $50 \mathrm{mM}$ sodium phosphate and $0.5 \mathrm{mM}$ DTT. Far-UV circular dichroism (CD) spectra were recorded on a Jasco J-720 spectropolarimeter at $25^{\circ} \mathrm{C}$ with a resolution of $0.5 \mathrm{~nm}$. Final spectra were the sum of four scans accumulated at a speed of 20 $\mathrm{nm} / \mathrm{min}$ with a response time of 1 second. Secondary structure was predicted using combined results of CONTINLL, SELCON3, and CDSSTR programs, CDPro Suite (http://lamar.colostate.edu/ sreeram/CDPro/main.html). 
Chromatin immunoprecipitation. Chromatin immunoprecipitation used a commercially available kit (Upstate Biotechnology Inc., Lake Placid, New York, USA) as described (23). Cells (10T1/2) were fixed in $1 \%$ formaldehyde, for 15 minutes at $37^{\circ} \mathrm{C}$ and quenched in $0.125 \mathrm{M}$ glycine. After lysis in SDS buffer containing $1 \%$ protease inhibitor cocktail (SigmaAldrich) and $1 \mathrm{mM}$ PMSF (Sigma-Aldrich), lysates were sonicated, cleared, and incubated overnight at $4^{\circ} \mathrm{C}$ in chromatin immunoprecipitation buffer containing $1 \%$ protease inhibitors and $1 \mathrm{mM}$ PMSF with $5 \mu \mathrm{l}$ of antiacetylated histone $\mathrm{H} 4$ or $\mathrm{H} 3 \mathrm{Ab}$ (Upstate Biotechnology Inc.). Immunocomplexes were recovered with $60 \mu \mathrm{l}$ salmon sperm DNA and protein Aagarose, incubated at $4^{\circ} \mathrm{C}$ for 1 hour, eluted, and heated to $65^{\circ} \mathrm{C}$ for 6 hours. DNA was recovered using proteinase $\mathrm{K}$, phenol-chloroform extraction, and ethanol precipitation. PCR was performed with primers to the SM $22 \alpha$ promoter CArG-containing region (24) and primers 5'-GGTCCTGCCCATAAAAGGTTT- $3^{\prime}$ and $5^{\prime}$-TGCCCATGGAAGTCTGCTTGG-3') (25). Immunoprecipitation and Western blot analysis. The $293 \mathrm{~T}$ cells were transfected and incubated for 48 hours. After cross-linking and quenching, the cells were scraped and lysed with lysis buffer $(20 \mathrm{mmol} / 1$ Tris, $\mathrm{pH}$ $8.0,150 \mathrm{mmol} / 1 \mathrm{NaCl}, 1 \%$ Igepal CA $630,0.5 \%$ sodium deoxycholate, $0.1 \%$ SDS, $2.5 \mathrm{mmol} / 1$ EDTA, 2.5 $\mathrm{mmol} / \mathrm{l}$ EGTA, $1 \%$ protease inhibitor cocktail, and 1 mM PMSF). After preclearing, $6 \mu \mathrm{l}$ anti-myc Ab (9E10; Sigma-Aldrich) was added, incubated at $4^{\circ} \mathrm{C}$ overnight, and the immunocomplex was recovered by 1 -hour incubation with $60 \mu \mathrm{l}$ protein A/G Sepharose beads (Santa Cruz Biotechnology Inc., Santa Cruz, California, USA). Endogenous HDAC1-8 were detected with specific Ab's (Zymed Laboratories Inc., South San Francisco, California, USA). For HDAC9, pcDNA3.1HDAC9 was cotransfected and detected with anti-V5 $\mathrm{Ab}$ (Invitrogen Life Technologies).

HDAC activity assay. HDAC activity was measured with colorimetric HDAC assay kit (Abcam, Cambridge, United Kingdom) as directed, with the following modifications. Transfected Cos cells were harvested and sonicated in buffer $(150 \mathrm{mM} \mathrm{NaCl}, 50$ $\mathrm{mM}$ Tris- $\mathrm{HCl}, \mathrm{pH} 8.0,1 \%$ Igepal CA 630) and precleared with $30 \mu \mathrm{l}$ protein A/G Sepharose beads (Santa Cruz Biotechnology Inc.). The supernatants were incubated with anti-myc $\mathrm{Ab}$ at $4{ }^{\circ} \mathrm{C}$ overnight, and $60 \mu \mathrm{l}$ protein $\mathrm{A} / \mathrm{G}$ Sepharose beads were added for 1 hour. After washing, the beads were equilibrated with $1 \times$ HDAC assay buffer, resuspended in $80 \mu \mathrm{l}$ HDAC assay buffer, and transferred to 96-well plates. Five microliters HDAC substrate, Boc-Lys(Ac)-pNA, was added and incubated for 1 hour at $37^{\circ} \mathrm{C}$. Ten microliters lysine developer was added, incubated for 30 minutes, and the absorbance at $405 \mathrm{~nm}$ was measured. An equal amount of protein A/G Sepharose was washed with lysis buffer, equilibrated with $1 \times$ HDAC assay buffer, and used to produce a standard curve by adding 0-100 $\mu \mathrm{mol} / 1$ deacetylated Boc-lys-pNA. The absorbance of samples is expressed as arbitrary units equivalent to the absorbance obtained with specific concentrations (micromoles per liter) of deacetylated standard. Transgenic or wild-type hearts were pooled, homogenized in lysis buffer, sonicated, and cleared by centrifugation. Twelve milligrams of protein was subjected to HDAC assay using $8 \mu \mathrm{l}$ anti-HA $\mathrm{Ab}$ (12CA5; Roche Diagnostics Corp.).

\section{Results}

Overexpression of Hop causes cardiac bypertrophy. We created transgenic mice in which Hop is expressed in the heart under the control of the $\alpha$-MHC promoter (26). This promoter is activated predominantly in the postnatal myocardium. In three independent lines of
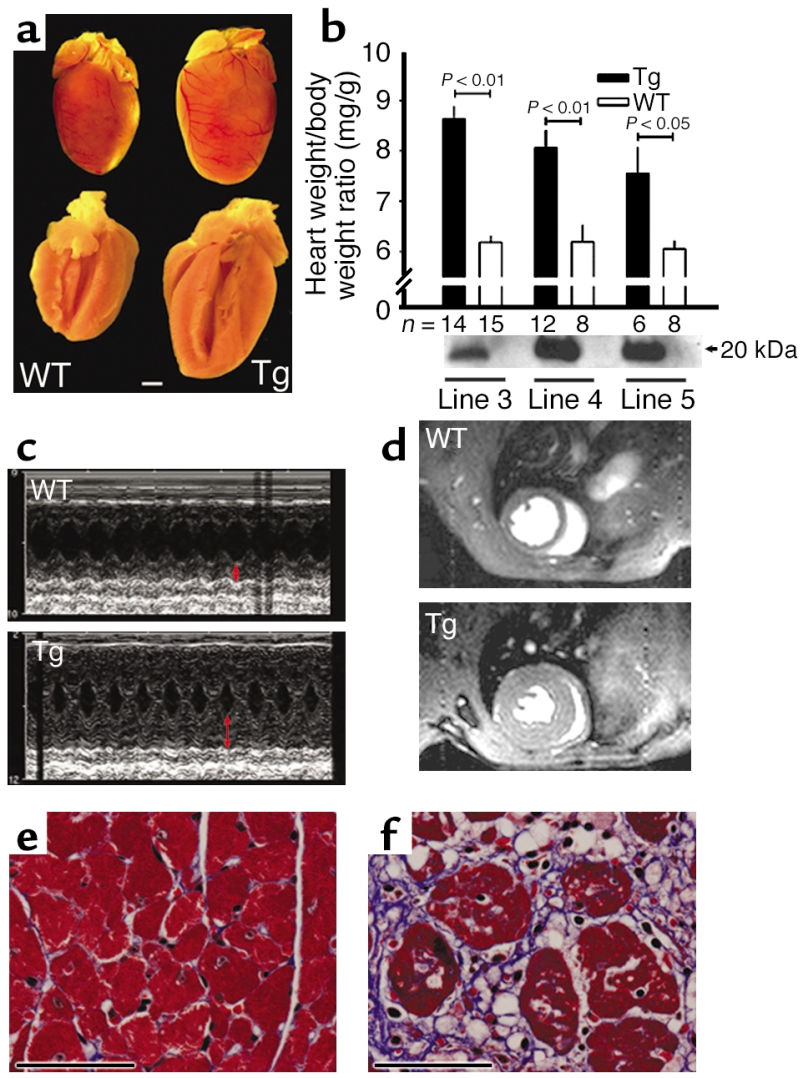

\section{Figure 1}

Transgenic expression of Hop in the heart causes cardiac hypertrophy. (a) Wild-type (left) and Hop transgenic ( $\mathrm{Tg}$ ) hearts at 8 weeks of age are shown. Scale bar: $1 \mathrm{~mm}$. (b) Heart weight-to-body weight ratios of Hop transgenic (black bars) and wild-type littermate (white bars) mice between 5 and 10 weeks of age are shown. Hop transgenic hearts are significantly enlarged. (c) M-mode echocardiography demonstrates thickened myocardial walls in transgenic mice (red arrows) and hyperdynamic function with cavity obliteration. (d) EKG-gated cardiac MRI reveals cardiac hypertrophy. Short-axis view images were captured during end diastole showing high contrast between blood (appearing bright) and myocardium. (e) Mason's trichrome staining of myocardium from wild-type 28-week-old mouse reveals normal cardiac histology. (f) Identical staining technique and magnification of Hop transgenic littermate reveals massive myocyte hypertrophy and significant interstitial fibrosis (blue). (e and f) Scale bars: $50 \mu \mathrm{m}$. 
a

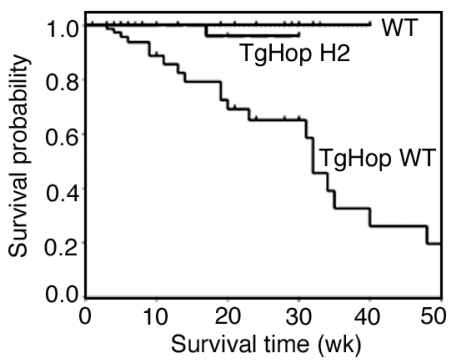

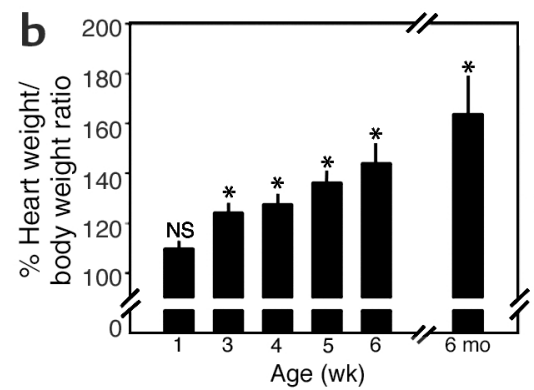
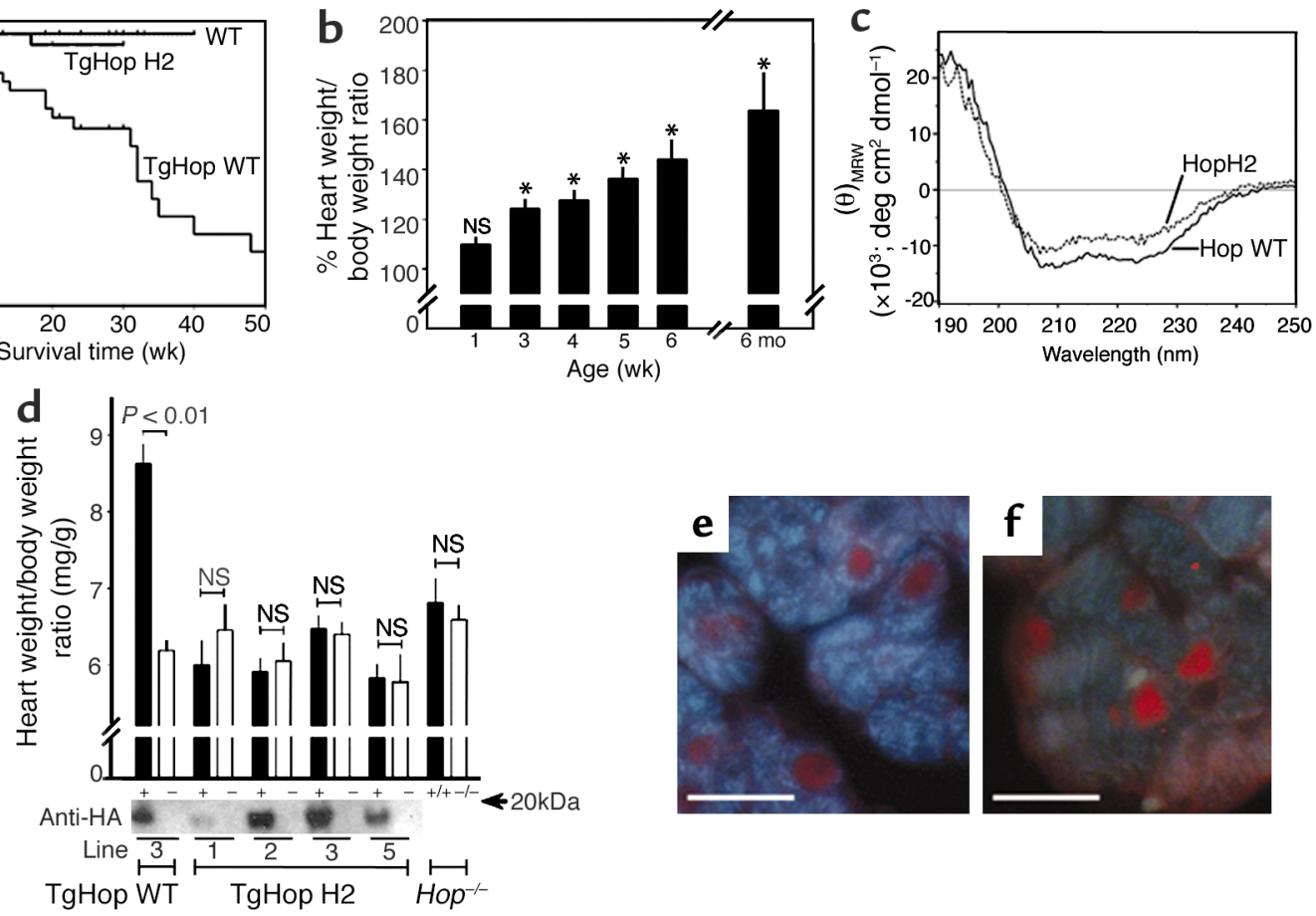

TgHop W

\section{Figure 2}

Early lethality and cardiac hypertrophy induced by Hop, but not by mutant $\mathrm{HopH}$ 2. (a) Kaplan-Meier plot revealing reduced survival of Hop transgenic mice (TgHop wt) compared with wild-type and HopH2 transgenic mice ( $\mathrm{TgHop} \mathrm{H} 2)$. (b) Cardiac hypertrophy in Hop transgenic mice is progressive. Heart weight-to-body weight ratios were calculated at various ages, as indicated, and expressed as percentage of change compared with wild-type littermates. Values from two independent transgenic lines are pooled and each bar represents the average of 8-16 data points. Error bars represent SEM. ${ }^{*} P<0.05$ compared with wild type. (c) Circular dichroism analysis of Hop and $\mathrm{HopH} 2 \mathrm{protein}$ indicates similar conformations and $\alpha$ helicity of wild-type Hop and $\mathrm{HopH} 2$ mutant proteins. $(\theta)_{\mathrm{MRW}}$, mean residue weight ellipticity. (d). Heart weight-to-body weight ratios of Hop transgenic mice, four independent lines of HopH2 transgenic mice, and Hop knockout mice between 4 and 8 weeks of age. Between 10 and 25 animals were assayed for each condition. No significant difference between transgenic and nontransgenic littermates was determined for $\mathrm{HopH} 2$ mice or for $\mathrm{Hop}^{-/-}$mice. Western blot analysis of heart tissue using anti-hemagglutinin (HA) antibody revealing expression of transgenic protein is also shown. Line numbers refer to independent transgenic lines. (e) Immunohistochemistry identifies nuclear epitope-tagged Hop protein in transgenic hearts (red). (f) HopH2 is also nuclear localized in transgenic hearts. Scale bars in e and f: $20 \mu \mathrm{m}$.

transgenic mice, we documented significant cardiac hypertrophy as early as 3 weeks after birth (Figure 1, $a$ and $b$ ). Transgenic Hop was expressed in these mice at two to tenfold endogenous levels as determined by Western blot analysis for wild-type and epitopetagged transgenic protein and by immunohistochemistry (data not shown). The heart weight-tobody weight ratio was increased approximately $25 \%$, and the ventricular walls were symmetrically thickened (Figure 1, $c$ and d) with hypertrophied myocytes (Figure 1, e and f). Invasive hemodynamic monitoring of 5- to 6-week-old animals revealed elevation of left ventricular end diastolic pressure in transgenic mice when compared with wild-type littermates $(5 \pm 1$ $\mathrm{mmHg}$ versus $2 \pm 0.3 \mathrm{mmHg}, P<0.01$ ) and impaired diastolic relaxation with no change in systolic blood pressure. Transgenic mice died suddenly and were found to have enlarged hearts with myocardial fibrosis (Figure 1f). Kaplan-Meier analysis revealed premature lethality with mean survival of 32 weeks, significantly different from that of control littermates (Figure 2a). Echocardiography and EKG-gated MRIs revealed severe concentric left and right ventricular hypertrophy in transgenic animals (Figure 1, c and d) that progressed variably to cardiac dilatation accompanied by heart failure. Electron microscopy revealed evidence of nonapoptotic cell death and mitochondrial swelling. All transgenic mice from these lines sacrificed after 4 weeks of age had enlarged hearts, and hypertrophy was progressive (Figure 2b). Quantitative RT-PCR revealed elevated levels of ANF and $\beta$-myosin heavy chain transcripts, genes commonly activated in the setting of hypertrophy.

Overexpression of a mutant form of Hop does not cause hypertrophy. Overexpression of a variety of proteins in cardiac myocytes can result in heart failure. In some cases this response is likely to be nonspecific. For instance, high-level expression of GFP under the control of the $\alpha$-MHC promoter in the heart has been shown to cause dilated cardiomyopathy (27). Therefore, we created transgenic mice that expressed a mutant form of Hop that we have shown previously to be devoid of transcriptional repression activity and that is unable to interact with SRF (19). This mutant form 
of Hop, designated HopH2, contains four altered amino acids in the second $\alpha$ helix of Hop. Both Hop and $\mathrm{HopH} 2$ are localized to the nucleus in transfected Cos cells (data not shown). Circular dichroism analysis of purified Hop and HopH2 protein produced in bacteria indicates that both proteins contain significant amounts of $\alpha$ helical content (Figure 2c) consistent with computer-based structural predictions and homology to the helix-turn-helix motif of homeodomain proteins. The $\alpha$-helical content of $\mathrm{HopH} 2$ is slightly less than that of Hop, consistent with mild disruption of the second $\alpha$-helix produced by the engineered amino acid substitutions.

We analyzed four independent transgenic lines of $\mathrm{HopH} 2$ mice. These lines produced varying levels of transgenic protein (Figure $2 \mathrm{~d}$ ). In several lines, these levels matched or exceeded the level of protein expression seen in hypertrophic Hop transgenic mice. Hearts in $\mathrm{HopH} 2$ mice, however, were not enlarged, and heart weight-to-body weight ratios were normal (Figure 2d). No cases of premature death were observed, and cardiac histology was normal without evidence of fibrosis (data not shown). Both wild-type Hop and HopH2 proteins were localized to the nucleus in vivo (Figure 2, e and $\mathrm{f}$ ). Hence, cardiac hypertrophy produced by overexpression of Hop is specific and is not reproduced by equivalent overexpression of a modified protein that is unable to inhibit SRF-dependent transcription.

Adult $\mathrm{Hop}^{-/-}$mice do not display bypertrophy. Alterations in cardiac size and in heart weight-to-body weight ratios have been described in Hop-deficient mice (20). Some $\mathrm{Hop}^{-/-}$embryos ( $\left.50 \%\right)$ succumb during midgestation with poorly developed myocardium, whereas other Hop-deficient mice display increased numbers of myocytes in the perinatal period. We have found that $\mathrm{Hop}^{-/-}$mice that survive to 4 weeks of age, however, have normal cardiac size and function. We examined heart weight-to-body weight ratios in $\mathrm{Hop}^{-/-}$mice between 4 and 8 weeks of life and found no difference from wild-type littermates (Figure 2d). Histology, echocardiography, and MRI analysis appeared normal (data not shown). In addition, we performed extensive invasive hemodynamic studies in $\mathrm{Hop}^{-/-}$mice at 40-45 weeks of life. We found no difference between $\mathrm{Hop}^{-/-}$mice and wildtype littermates in terms of blood pressure, heart rate, left ventricular end diastolic pressure, contractility, or relaxation, with or without isoproterenol infusion (Tables 1 and 2). Hence, cardiac hypertrophy produced by overexpression of Hop in transgenic mice is a gain-of-function phenotype and is not reproduced by loss of function in knockout mice.

HDAC inhibitors prevent Hop-induced repression of transcription. We investigated the mechanism by which Hop acts to inhibit SRF-dependent transcription by testing chemical inhibitors of histone deacetylase activity in cell-based transcription assays. Histone modification through acetylation and/or methylation is a common mechanism for regulation of global gene expression.
Cotransfection of a reporter construct containing two consensus SRF-binding sites derived from the previously described SM $22 \alpha$ promoter with a myocardin expression vector in Cos cells results in several hundred-fold activation of luciferase activity compared
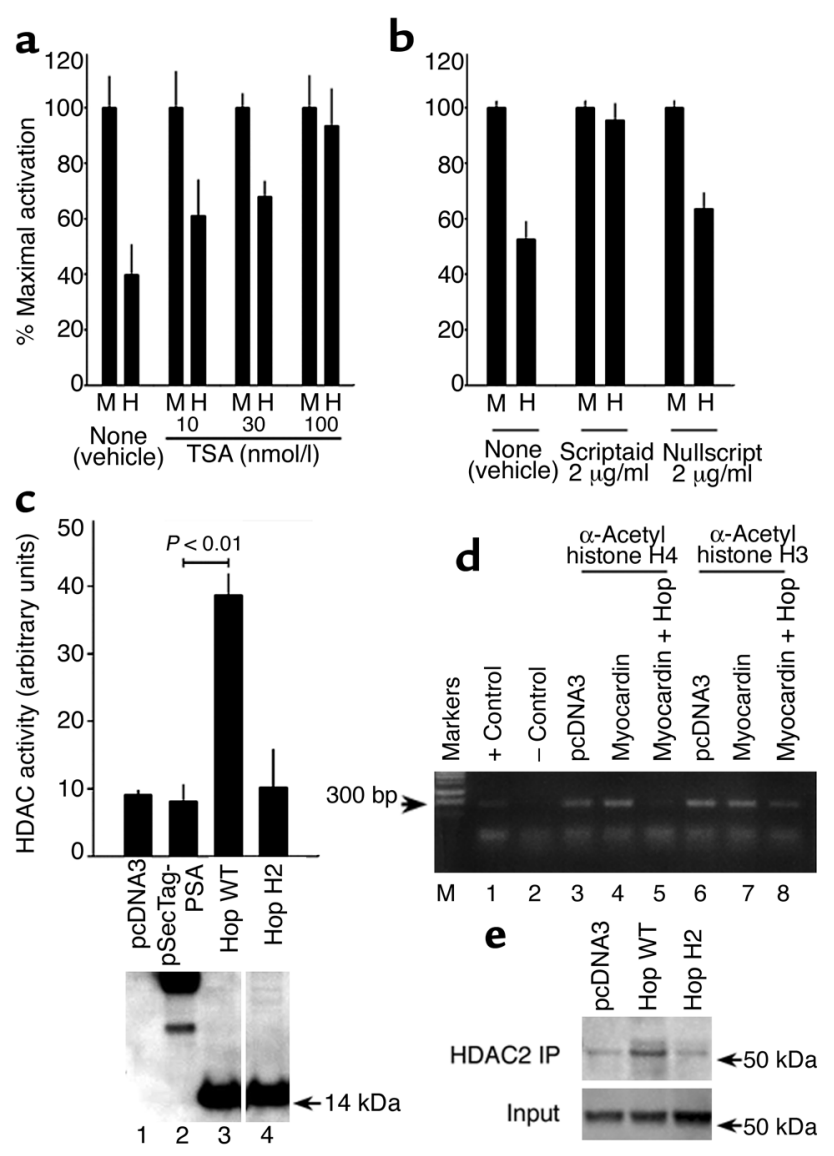

Figure 3

Hop recruits HDAC activity to repress transcription. (a) Cos cells were transfected with a luciferase reporter plasmid including regulatory elements from the SM22 $\alpha$ promoter with binding sites for SRF. Myocardin cotransfection induces dramatic activation assigned a value of $100 \%$ maximal activation (M). Cotransfection of myocardin and $\mathrm{Hop}(\mathrm{H})$ results in inhibition of myocardin-induced activation. The ability of Hop to inhibit myocardin-induced activation is attenuated in a dose-dependent fashion by TSA. (b) Hop inhibitory activity is also blocked by Scriptaid but not by Nullscript. (c) Immunoprecipitation of Hop from transfected Cos cells results in precipitation of HDAC activity. Cells were transfected with vector alone (pcDNA3) or myc-tagged control (pSecTag-PSA), Hop, or $\mathrm{HopH} 2$, followed by immunoprecipitation with anti-myc Ab. HDAC activity in the pellet was assayed and reported as arbitrary units (see Methods). Western blot analysis with anti-myc Ab of input material used for immunoprecipitation is shown below. (d) Chromatin immunoprecipitation reveals decrease in acetylated histones after Hop expression. The 10T1/2 cells were transfected as in a, followed by chromatin immunoprecipitation with Ab's to acetylated histone $\mathrm{H} 4$ or $\mathrm{H} 3$ and detection of SM $22 \alpha$ promoter fragments by PCR in precipitate. (e) Coimmunoprecipitation of HDAC2 and Hop. The 293T cells were transfected with control vector (pcDNA3), Hop, or $\mathrm{HopH} 2$, followed by immunoprecipitation and Western blot analysis for HDAC2. A Western blot using anti-HDAC2 Ab of the cell extracts used in each case is shown (Input). 
Table 1

Cardiac morphometric and baseline hemodynamic variables in Hop knockout and wild-type littermate mice

\begin{tabular}{lccc}
\hline & $H o p^{-/-}(n=10)$ & Wild-type $(n=12)$ & $P$ value \\
Heart weight $(\mathrm{mg})$ & $153 \pm 8$ & $170 \pm 8$ & 0.17 \\
Heart weight/body weight $(\mathrm{mg} / \mathrm{g})$ & $4.4 \pm 0.2$ & $4.6 \pm 0.4$ & 0.43 \\
Heart rate $\left(\mathrm{min}^{-1}\right)$ & $508 \pm 19$ & $520 \pm 14$ & 0.61 \\
Systolic aortic pressure $(\mathrm{mmHg})$ & $94 \pm 7$ & $88 \pm 4$ & 0.41 \\
Left ventricular systolic pressure $(\mathrm{mmHg})$ & $91 \pm 6$ & $1.0 \pm 0.5$ & 0.70 \\
Left ventricular end diastolic pressure $(\mathrm{mmHg})$ & $1.9 \pm 0.6$ & $8,399 \pm 574$ & 0.15 \\
Peak positive dP/dt $(\mathrm{mmHg} / \mathrm{s})$ & $8,202 \pm 1108$ & $-7,441 \pm 598$ \\
Peak negative $\mathrm{dP} / \mathrm{dt}(\mathrm{mmHg} / \mathrm{s})$ & $-7,031 \pm 756$ & 0.88 \\
\hline
\end{tabular}

Data are shown as mean \pm SEM. $d P / d t$, change in ventricular pressure with respect to time.

with cells transfected with the luciferase reporter construct and control vectors. Myocardin binds directly to endogenous SRF, which is present in Cos cells and is responsible for this potent transactivation (28). Addition of Hop markedly reduces myocardin and SRFdependent activation $(19,20)$. Importantly, the ability of Hop to inhibit myocardin-dependent transcriptional activation is severely attenuated in a dosedependent fashion by TSA, which functions by inhibiting HDAC activity (Figure 3a) (29). TSA (100 $\mathrm{nM}$ ) is sufficient to completely inhibit Hop activity in this assay. Likewise, Scriptaid $(2 \mu \mathrm{g} / \mathrm{ml})$, an alternate HDAC inhibitor, is able to completely block Hop activity. Nullscript, a chemically modified version of Scriptaid that is devoid of HDAC-blocking activity, has no effect on Hop-mediated repression (Figure 3b). Hence, the ability of Hop to repress SRF-dependent transcription requires HDAC activity.

Hop recruits $H D A C$ activity. To determine if Hop can contribute to a complex that includes HDAC activity, we transfected Cos cells with Hop, a mutant form of Hop (HopH2) that fails to repress transcription, or with control vectors. We immunoprecipitated wildtype or mutant Hop and assayed the precipitate for HDAC activity. Wild-type Hop is able to precipitate significant HDAC activity, while a control protein (epitope-tagged prostate-specific antigen, PSA) expressed at equal levels does not (Figure 3c). HopH2, which fails to repress transcription, is also unable to precipitate HDAC activity (Figure 3c). Hence, the ability of Hop to repress transcription correlates with the ability to form a complex that includes HDAC activity.

We used chromatin immunoprecipitation to determine the level of histone acetylation of the SM22 $\alpha 5^{\prime}$ flanking region in the presence or absence of Hop. We used an $\mathrm{Ab}$ specific for acetylated histone $\mathrm{H} 3$ and a distinct $\mathrm{Ab}$ specific for acetylated histone $\mathrm{H} 4$. Transfection of myocardin, which is associated with marked activation of the SM $22 \alpha$ promoter, is associated with the presence of acetylated histone $\mathrm{H} 3$ and $\mathrm{H} 4$, which allows for precipitation of the SM22 $\alpha$ upstream region (Figure 3d). Addition of Hop results in a marked decrease in acetylated histone $\mathrm{H} 3$ and $\mathrm{H} 4$ (Figure 3d). Hop also reduced the basal amount of acetylated histones at the SM22 $\alpha$ promoter in the absence of myocardin (data not shown). These results are consistent with the ability of Hop to recruit HDAC activity resulting in histone deacetylation and transcriptional repression. Immunoprecipitation followed by Western blot analysis revealed that Hop forms a complex with HDAC2 (Figure 3e), while the mutant form of Hop, $\mathrm{HopH} 2$, does not. HDAC2 is expressed in the murine heart (data not shown). Hop was also able to interact to a lesser degree with HDAC3, but we did not detect interactions with HDAC1 or HDAC4-HDAC9 (data not shown). Coimmunoprecipitation studies were per-

\section{Table 2}

Hemodynamic response to isoproterenol in Hop knockout and wild-type littermate mice

\begin{tabular}{|c|c|c|c|}
\hline & Baseline & Maximum isoproterenol response & $\%$ Change \\
\hline \multicolumn{4}{|l|}{$\mathrm{Hop}^{-/-}$mice $(n=6)$} \\
\hline Heart rate $\left(\mathrm{min}^{-1}\right)$ & $511 \pm 16$ & $562 \pm 6^{A}$ & $11 \pm 3^{\mathrm{B}}$ \\
\hline Peak positive $\mathrm{dP} / \mathrm{dt}(\mathrm{mmHg} / \mathrm{s})$ & $8,319 \pm 1,154$ & $10,828 \pm 1204^{A}$ & $35 \pm 11^{B}$ \\
\hline Peak negative $\mathrm{dP} / \mathrm{dt}(\mathrm{mmHg} / \mathrm{s})$ & $-8,315 \pm 876$ & $-9,885 \pm 1,073$ & $22 \pm 11^{\mathrm{B}}$ \\
\hline \multicolumn{4}{|l|}{ Wild-type mice $(n=11)$} \\
\hline Heart rate $\left(\mathrm{min}^{-1}\right)$ & $509 \pm 21$ & $578 \pm 17^{A}$ & $15 \pm 4$ \\
\hline Peak positive $\mathrm{dP} / \mathrm{dt}(\mathrm{mmHg} / \mathrm{s})$ & $7,753 \pm 472$ & $11,044 \pm 887^{A}$ & $42 \pm 7$ \\
\hline Peak negative $\mathrm{dP} / \mathrm{dt}(\mathrm{mmHg} / \mathrm{s})$ & $-7,894 \pm 630$ & $-8,688 \pm 907$ & $10 \pm 6$ \\
\hline
\end{tabular}

Data are shown as mean \pm SEM. ${ }^{A} P<0.05$ versus baseline. ${ }^{B}$ No significant difference for comparison of percentage of change for each variable in $H_{0 p} / /$ and wild-type mice. $\mathrm{dP} / \mathrm{dt}$, change in ventricular pressure with respect to time. 
formed using extracts of cultured cells, and it remains unknown if the interaction between Hop and HDAC2 is direct or if additional components of the HopHDAC complex exist.

HDAC inbibition prevents bypertrophy. In transgenic mouse hearts, Hop can also interact with endogenous proteins that exhibit HDAC activity. Precipitation of epitope-tagged Hop from the hearts of transgenic mice resulted in coprecipitation of HDAC activity (Figure 4a). To determine if cardiac hypertrophy in Hop transgenic mice is the result of HDAC-mediated transcriptional repression, we treated 3- to 5-week-old wild-type and Hop transgenic littermates with TSA. TSA, delivered by daily intraperitoneal injection at a dose of 0.6 $\mathrm{mg} / \mathrm{kg}$ body weight (a dose sufficient to inhibit endogenous HDAC activity) (29, 30), was well tolerated and did not affect overall body weight or heart weight-to-body weight ratios in wild-type mice compared with those injected with vehicle alone (Figure $4 b$ ).
In Hop-transgenic mice, TSA resulted in a significant reduction in heart weight-to-body weight ratio compared with vehicle-treated littermates. We also treated Hop transgenic mice with sodium valproate, an unrelated medication with HDAC inhibitor activity (31). Cardiac hypertrophy, as assessed by heart weight-tobody weight ratio, was significantly attenuated by valproate (Figure 4c). Thus, Hop-induced, HDAC-dependent, transcriptional repression induces cardiac hypertrophy, and this process is attenuated by therapeutic delivery of chemical HDAC inhibitors.

To determine if inhibition of HDAC activity might prevent cardiac hypertrophy induced by alternative mechanisms, we treated wild-type CD1 mice with isoproterenol infusion (30 mg/kg/day) using an implantable delivery system for a 2 -week period (32-34). This treatment resulted in significant cardiac hypertrophy as assessed by MRI (not shown) and by heart weight-to-body weight ratio (Figure 4d). Concomitant
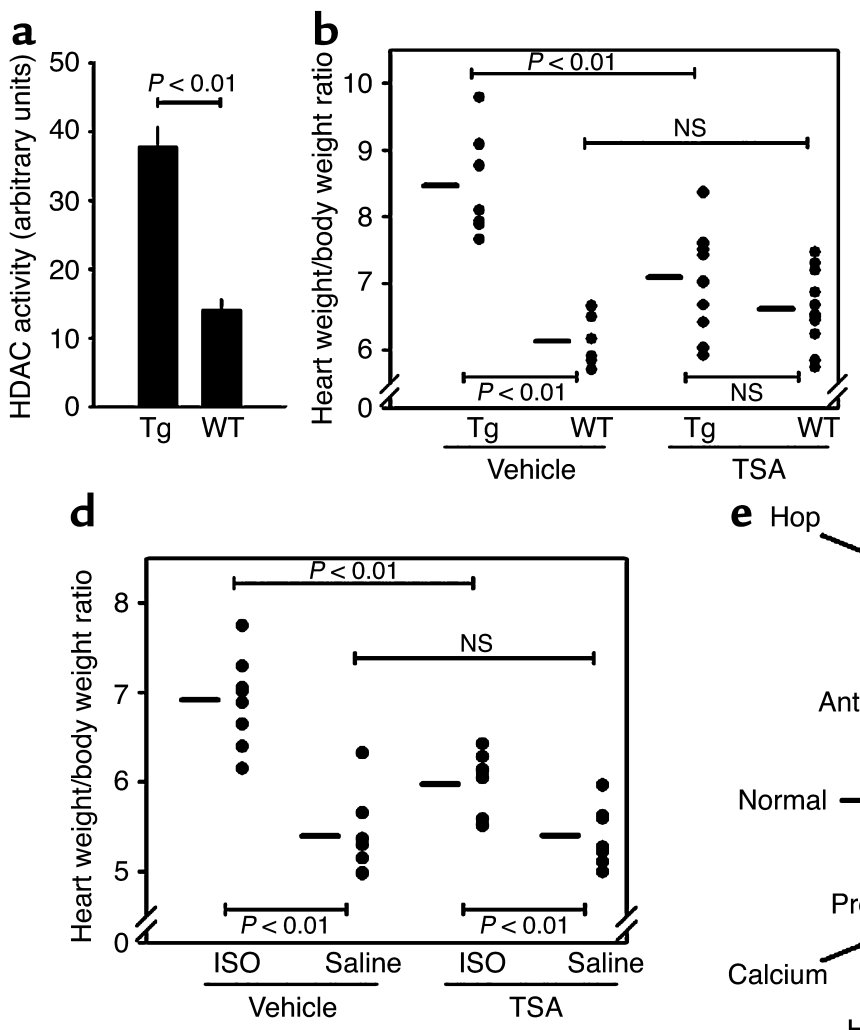

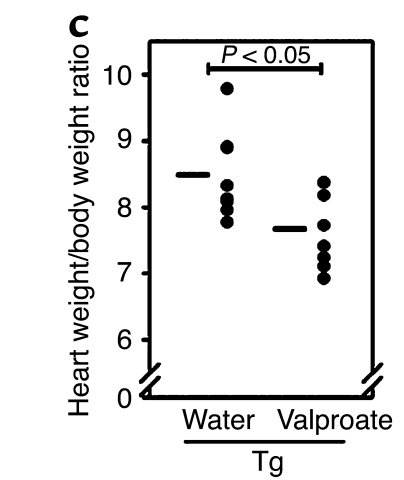

e Hop

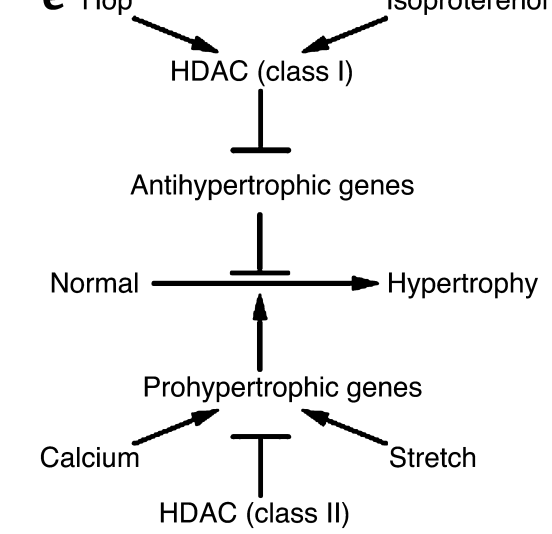

\section{Figure 4}

Treatment with HDAC inhibitor prevents cardiac hypertrophy in Hop transgenic mice. (a) HDAC activity was associated with immunoprecipitated transgenic Hop protein derived from hearts of transgenic Hop mice (5-6 weeks of age) when compared with wild-type littermates. (b) Transgenic and wild-type littermates were treated with daily injections of TSA between the ages of 3 and 5 weeks. Heart weight-to-body weight ratios were calculated at 5 weeks. Bars indicate mean of values shown, and each filled circle represents one animal. TSA significantly attenuated cardiac hypertrophy, but did not affect heart weight-to-body weight ratio in nontransgenic mice. (c) Hop transgenic mice were also treated with sodium valproate between 3 and 5 weeks of age, which resulted in attenuation of cardiac enlargement at 5 weeks. (d) Wild-type mice were treated with isoproterenol (ISO) infusion between 3 and 5 weeks of life, with or without daily injection of TSA. Heart weight-to-body weight ratio increased significantly with isoproterenol, and this increase was significantly inhibited by TSA. (e) Model depicting the ability of Hop or other hypertrophic stimuli to recruit class I HDACs resulting in inhibition of antihypertrophic gene programs. Inducers of cardiac hypertrophy are also thought to function by altering calcium homeostasis or mechanical stretch and induce prohypertrophic genes. Class II HDACs can inhibit some prohypertrophic programs. The relative balance of pro- and antihypertrophic gene programs will determine the extent of myocyte hypertrophy. 
treatment with daily TSA injections significantly abrogated this increase in heart weight-to-body weight ratio (Figure 4d), suggesting that some forms of cardiac hypertrophy unrelated to transgenic overexpression of Hop can be blocked by inhibition of HDAC activity.

\section{Discussion}

These studies demonstrate the existence of a previously unrecognized antihypertrophic transcriptional program that functions in cardiac myocytes. Overexpression of Hop in adult mice functions to repress this program, thus shifting the balance between proand antihypertrophic pathways (Figure 4e). While numerous stimuli have been identified that induce hypertrophy in animal models and in humans $(6,8$, $9,35)$, generally these have been thought to manifest pathological effects by inducing prohypertrophic gene expression. For instance, alterations in calcium handling can result in activation of calcineurin and subsequent activation of gene expression dependent upon NFAT (10). Also, MEF2 activation of gene expression correlates with cardiac hypertrophy in some animal models. Importantly, class II HDACs can inhibit MEF2 activity and thus may antagonize prohypertrophic gene expression $(12,36,37)$ (Figure $4 \mathrm{e})$. Our results suggest that homeostatic maintenance of cardiomyocyte size is also dependent upon the expression of antihypertrophic genes and that this expression can be inhibited by Hop in association with the class I HDAC, HDAC2. Hence, the overall effect of HDAC activity and of HDAC inhibition is likely to depend upon the relative balance of proand antihypertrophic gene programs that are modified by specific recruitment of individual complexes to unique sets of genes.

In cultured cardiac myocytes, HDAC inhibition prevents the induction of fetal gene programs induced by hypertrophic stimulants such as phenylephrine and endothelin-1 (38). Hence, the predominant effect of nonspecific HDAC inhibition on cultured myocytes and in our transgenic and isoproterenol-treated mice is to prevent cardiac hypertrophy. This is an unexpected result, given the fact that inactivation of HDAC9, a class II HDAC, by homologous recombination in the mouse, resulted in animals unusually sensitive to hypertrophic stimuli (12).

While Hop can affect SRF activity in cultured cells and in vivo, it remains unclear what SRF-independent functions of Hop remain to be elucidated. Interestingly, overexpression of SRF in transgenic mice causes cardiac hypertrophy (39), while overexpression of a mutant form of SRF that does not bind DNA results in rapid postnatal demise and a poorly developed myocardium (40). These data suggest that Hop overexpression may affect only a subset of SRFdependent pathways or may have effects in addition to those mediated by SRF. Previous studies investigating the role of Hop during embryonic development implicated a role for Hop in the modulation of
SRF-dependent transcription $(19,20)$, but alternative or additional functions for Hop in embryonic or adult animals may also exist.

Previous studies have focused on the identification of genetic pathways that are activated during hypertrophy and induce cardiac growth. Our studies highlight the importance of previously unappreciated molecular programs that normally function to repress hypertrophic responses. The full program of genes repressed by the Hop-HDAC2-containing complex remains to be defined. Microarray analysis of Hop transgenic hearts, however, indicates repression of $c$-fos, a classic SRF transcriptional target (41), consistent with previous data indicating that Hop can repress SRF-dependent transcription $(19,20)$. Interestingly, we also identified an RNA helicase, Dby, that is consistently downregulated in Hop transgenic hearts. A related DEAD box containing RNA helicase, CHAMP, is able to repress phenylephrine-induced myocyte hypertrophy in cultured neonatal cardiac myocytes (42).

HDAC inhibitors are already in clinical trials for a variety of noncardiac disorders and could potentially influence normal or pathological cardiac function $(17,18,43,44)$. The identification and further elucidation of antihypertrophic transcriptional pathways will offer novel therapeutic targets for the treatment of congestive heart failure.

\section{Acknowledgments}

This work was supported by NIH grants HL-61475 and HL-071546 to J.A. Epstein. H. Kook's work was supported partly by the Basic Research Program of the Korea Science and Engineering Foundation (R13-2002013-01000-0). A. Gitler is supported by an NIH predoctoral training grant in developmental biology to the University of Pennsylvania. Philadelphia: W.B. Saunders. ch. 13.

2. Marks, A.R. 2003. Calcium and the heart: a question of life and death. J. Clin. Invest. 111:597-600. doi:10.1172/JCI200318067.

3. Epstein, N.D., and Davis, J.S. 2003. Sensing stretch is fundamental. Cell. 112: $147-150$

4. McKinsey, T.A., and Olson, E.N. 1999. Cardiac hypertrophy: sorting out the circuitry. Curr. Opin. Genet. Dev. 9:267-274.

5. Braunwald, E. 1997. Shattuck lecture-cardiovascular medicine at the turn of the millennium: triumphs, concerns, and opportunities. N. Engl. J. Med. 337:1360-1369.

6. Seidman, J.G., and Seidman, C. 2001. The genetic basis for cardiomyopathy: from mutation identification to mechanistic paradigms. Cell. 104:557-567.

7. Rockman, H.A., Koch, W.J., and Lefkowitz, R.J. 1997. Cardiac function in genetically engineered mice with altered adrenergic receptor signaling. Am. J. Physiol. 272:H1553-H1559.

8. Knoll, R., et al. 2002. The cardiac mechanical stretch sensor machinery involves a $Z$ disc complex that is defective in a subset of human dilated cardiomyopathy. Cell. 111:943-955.

9. Hunter, J.J., and Chien, K.R. 1999. Signaling pathways for cardiac hypertrophy and failure. N. Engl. J. Med. 341:1276-1283.

10. Molkentin, J.D., et al. 1998. A calcineurin-dependent transcriptional pathway for cardiac hypertrophy. Cell. 93:215-228.

11. McKinsey, T.A., Zhang, C.L., and Olson, E.N. 2000. Activation of the myocyte enhancer factor- 2 transcription factor by calcium/calmodulindependent protein kinase-stimulated binding of 14-3-3 to histone deacetylase 5. Proc. Natl. Acad. Sci. U. S. A. 97:14400-14405.

12. Zhang, C.L., et al. 2002. Class II histone deacetylases act as signal-responsive repressors of cardiac hypertrophy. Cell. 110:479-488. 
13. Struhl, K. 1998. Histone acetylation and transcriptional regulatory mechanisms. Genes Dev. 12:599-606.

14. Jenuwein, T., and Allis, C.D. 2001. Translating the histone code. Science. 293:1074-1080

15. Fischle, W., Wang, Y., and Allis, C.D. 2003. Histone and chromatin crosstalk. Curr. Opin. Cell Biol. 15:172-183.

16. Minucci, S., Nervi, C., Lo Coco, F., and Pelicci, P.G. 2001. Histone deacetylases: a common molecular target for differentiation treatment of acute myeloid leukemias? Oncogene. 20:3110-3115.

17. Vigushin, D.M., and Coombes, R.C. 2002. Histone deacetylase inhibitors in cancer treatment. Anticancer Drugs. 13:1-13.

18. Kwon, H.J., Kim, M., and Kim, D.H. 2003. Histone deacetylase in carcinogenesis and its inhibitors as anti-cancer agents. J. Biochem. Mol. Biol. 36:110-119.

19. Chen, F., et al. 2002. Hop is an unusual homeobox gene that modulates cardiac development. Cell. 110:713-723.

20. Shin, C.H., et al. 2002. Modulation of cardiac growth and development by HOP, an unusual homeodomain protein. Cell. 110:725-735.

21. Gulick, J., Subramaniam, A., Neumann, J., and Robbins, J. 1991. Isolation and characterization of the mouse cardiac myosin heavy chain genes. J. Biol. Chem. 266:9180-9185.

22. Zhou, R., Pickup, S., Glickson, J., Scott, C., and Ferarri, V.A. 2003. Assessment of global and regional myocardial function in the mouse using cine and tagged MRI. Magn. Reson. Med. 49:760-764.

23. Kuwahara, K., et al. 2001. The neuron-restrictive silencer element-neuron-restrictive silencer factor system regulates basal and endothelin 1inducible atrial natriuretic peptide gene expression in ventricular myocytes. Mol. Cell Biol. 21:2085-2097.

24. Strobeck, M., et al. 2001. Binding of serum response factor to CArG box sequences is necessary but not sufficient to restrict gene expression to arterial smooth muscle cells. J. Biol. Chem. 276:16418-16424.

25. Qiu, P., and Li, L. 2002. Histone acetylation and recruitment of serum responsive factor and CREB-binding protein onto SM22 promoter during SM22 gene expression. Circ. Res. 90:858-865.

26. Palermo, J., Gulick, J., Colbert, M., Fewell, J., and Robbins, J. 1996. Transgenic remodeling of the contractile apparatus in the mammalian heart Circ. Res. 78:504-509.

27. Huang, W.Y., Aramburu, J., Douglas, P.S., and Izumo, S. 2000. Transgenic expression of green fluorescence protein can cause dilated cardiomyopathy. Nat. Med. 6:482-483.

28. Wang, D., et al. 2001. Activation of cardiac gene expression by myocardin, a transcriptional cofactor for serum response factor. Cell. 105:851-862.

29. Yoshida, M., Kijima, M., Akita, M., and Beppu, T. 1990. Potent and specific inhibition of mammalian histone deacetylase both in vivo and in vitro by trichostatin A. J. Biol. Chem. 265:17174-17179.

30. Nervi, C., et al. 2001. Inhibition of histone deacetylase activity by trichostatin A modulates gene expression during mouse embryogenesis without apparent toxicity. Cancer Res. 61:1247-1249.

31. Gottlicher, M., et al. 2001. Valproic acid defines a novel class of HDAC inhibitors inducing differentiation of transformed cells. EMBO J. 20:6969-6978.

32. Bueno, O.F., et al. 2002. Impaired cardiac hypertrophic response in calcineurin A beta-deficient mice. Proc. Natl. Acad. Sci. U. S. A. 99:4586-4591.

33. Friddle, C.J., Koga, T., Rubin, E.M., and Bristow, J. 2000. Expression profiling reveals distinct sets of genes altered during induction and regression of cardiac hypertrophy. Proc. Natl. Acad. Sci. U. S. A. 97:6745-6750.

34. Soonpaa, M.H., and Field, L.J. 1994. Assessment of cardiomyocyte DNA synthesis during hypertrophy in adult mice. Am. J. Physiol. 266:H1439-H1445.

35. Chien, K.R. 2000. Genomic circuits and the integrative biology of cardiac diseases. Nature. 407:227-232.

36. McKinsey, T.A., Zhang, C.L., and Olson, E.N. 2000. Activation of the myocyte enhancer factor- 2 transcription factor by calcium/calmodulindependent protein kinase-stimulated binding of 14-3-3 to histone deacetylase 5. Proc. Natl. Acad. Sci. U. S. A. 97:14400-14405

37. Passier, R., et al. 2000. CaM kinase signaling induces cardiac hypertrophy and activates the MEF2 transcription factor in vivo. J. Clin. Invest. 105:1395-1406.

38. Antos, C.L., et al. 2003. Dose-dependent blockade to cardiomyocyte hypertrophy by histone deacetylase inhibitors. J. Biol. Chem. 278:28930-28937.

39. Zhang, X., et al. 2001. Cardiomyopathy in transgenic mice with cardiacspecific overexpression of serum response factor. Am. J. Physiol. Heart Circ. Physiol. 280:H1782-H1792.

40. Zhang, X., et al. 2001. Early postnatal cardiac changes and premature death in transgenic mice overexpressing a mutant form of serum response factor. J. Biol. Chem. 276:40033-40040.

41. Norman, C., Runswick, M., Pollock, R., and Treisman, R. 1988. Isolation and properties of cDNA clones encoding SRF, a transcription factor that binds to the c-fos serum response element. Cell. 55:989-1003.

42. Liu, Z.P., and Olson, E.N. 2002. Suppression of proliferation and cardiomyocyte hypertrophy by CHAMP, a cardiac-specific RNA helicase. Proc. Natl. Acad. Sci. U. S. A. 99:2043-2048.

43. Petti, M.C., et al. 2002. Complete remission through blast cell differentiation in PLZF/RARalpha-positive acute promyelocytic leukemia: in vitro and in vivo studies. Blood. 100:1065-1067.

44. Fabian, C.J., and Kimler, B.F. 2001. Beyond tamoxifen new endpoints for breast cancer chemoprevention, new drugs for breast cancer prevention. Ann. N. Y. Acad. Sci. 952:44-59. 